\title{
A novel skeletal anchorage for rigid external distractor
}

\author{
Rodrigo Fariña ${ }^{1}$, Felipe Salinas ${ }^{2}$ \\ ${ }^{1}$ Department of Oral and Maxillofacial Surgery, Hospital del Salvador, Hospital San Borja Arriarán, Universidad de Chile, Santiago 7510052, Chile. \\ ${ }^{2}$ Department of Oral and Maxillofacial Surgery, Hospital San José de Melipilla, Santiago 9580000, Chile.
}

Correspondence to: Dr. Rodrigo Fariña, Department of Oral and Maxillofacial Surgery, Hospital del Salvador, Hospital San Borja Arriarán, Universidad de Chile, Providencia 2330, appt. 23, Santiago 7510052, Chile. E-mail: rofari@gmail.com

How to cite this article: Fariña R, Salinas F. A novel skeletal anchorage for rigid external distractor. Plast Aesthet Res 2017;4:144-9.

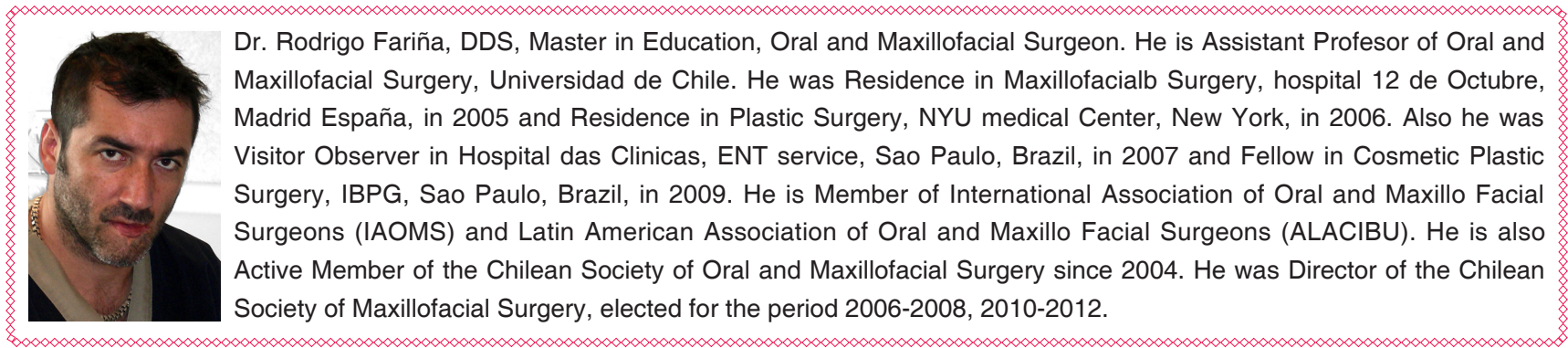

Article history:

Received: 10-07-2017

Accepted: 24-08-2017

Published: 05-09-2017

Key words:

Rigid external device, rigid external distractor, maxillary hypoplasia, maxillary retrusion

\begin{abstract}
Aim: Maxillofacial surgery has always aimed to find alternative therapies to treat severe maxillary hypoplasia. Distraction osteogenesis of the midface has become the technique with the best functional and aesthetic results. Nevertheless, anchoring a distractor to the middle third of the face continues to involve complex planning. Plus, achieving the desired force vector can sometimes be cumbersome and uncomfortable. The aim of this study is to propose a novel skeletal anchorage technique for the rigid external distractor. Methods: Non-controlled, prospective study of 9 patients with severe midface hypoplasia who were treated with distraction osteogenesis using a rigid external distractor anchored to the infraorbital rims and the bilateral pyriform apertures. The activation phase started the first postoperative day at a rate of $1 \mathrm{~mm}$ per day. The consolidation period lasted 6 to 8 weeks. Results: Eight patients achieved the desired distraction objective ( $24.5 \mathrm{~mm}$ on average), with only 1 suffering a 5-mm relapse. None of the patients reported complications. Conclusion: Distraction osteogenesis of the midface by skeletal anchorage is an alternative method when treating patients with severe maxillary hypoplasia. It has significant advantages compared to traditional anchoring because it simplifies the procedure, diminishes the costs and complications.
\end{abstract}




\section{INTRODUCTION}

Solving cases of severe maxillary hypoplasia has always been a challenge for oral and maxillofacial surgeons..$^{[1,2]}$ Molina and Ortiz-Monasterio ${ }^{[3]}$ proposed a gradual advancement of the maxilla using a facemask for elastic orthodontic traction or distraction. However, an adequate maxillary advancement in cases of severe hypoplasia was not achieved. Besides, the forces applied could not be controlled and ulcers in the chin and forehead were created. ${ }^{[4]}$ Polley and Figueroa $^{[1,5]}$ also used elastic distraction with facemask and had similar findings, reporting insufficient maxillary advancement (4 to $6 \mathrm{~mm}$ on average) with undercorrected residual inverted anterior bite.

In 1997, some authors proposed the use of the rigid external distraction device I (RED I) ${ }^{[4-6]}$ in order to overcome these shortcomings. The device was anchored to the skull and the denture in order to distract facial bones. ${ }^{[7]}$ The use of the RED I became an excellent treatment strategy, because it allowed precise and controlled distraction osteogenesis of the maxilla. ${ }^{[8,9]}$ Additionally, results were stable and predictable. ${ }^{[10]}$ Later, the RED II was introduced to improve the vector control by means of an additional anchorage to the zygomatic bone plate. All of these attributes make the use of RED I and II an excellent treatment alternative for patients with severe maxillary hypoplasia.

RED I and II use a custom-made intraoral orthodontic splint ${ }^{[1]}$ anchored to the first permanent molars or second temporary molars to generate the maxilla pulling force. The splint is made using cast models and 0.050 or $0.045 \mathrm{~mm}$ stainless steel wires. A wire emerges from the splint up to the height of the nostril floor, which generally coincides with the maxilla's centre of rotation.

Despite the many advantages offered by the RED I and II, both devices have some disadvantages. The need for teeth to anchor the device is one of them. ${ }^{[11]}$ Dental anchoring results in patient discomfort and interferes with the normal functions of the oral cavity, i.e. eating, speaking and performing proper oral hygiene. ${ }^{[12]}$ On the other hand, teeth act as an intermediary between the RED and the bone to be distracted, impeding direct force to be applied to the bone. ${ }^{[11]}$

The aim of this article is to propose an alternative to the dental anchorage used when distracting with RED I and II. We suggest the use of a skeletal anchorage with a gauge of 0.4 or $0.5 \mathrm{~mm}$ of stainless steel wire, that we called skeletal anchorage for the rigid external device (SARED).

\section{METHODS}

The study was a non-controlled, prospective study involving 9 patients, 3 female and 6 male ( 9 to 24 years old; mean age $17.5 \pm 5.4$ years), selected from the Department of Oral and Maxillofacial Surgery at Hospital del Salvador between April 2007 and January 2015. Inclusion criteria were patients diagnosed with severe midface hypoplasia aged 5 years or older (to allow proper post distraction oclusal stability and an adequate teeth interdigitation shown in the handarticulated models).

Preoperative assessment included clinical digital photography, articulated models and three-dimensional studies to plan a surgical treatment objective (STO).

Distraction osteogenesis of the midface was performed using SARED by means of the rigid external distraction device (RED, Cibei Medical Treatment Appliance Co. Ltd., Ningbo, China). Depending on the STO a Le Fort I or III osteotomy was done. When a Le Fort III osteotomy $(n=6)$ was performed, the RED was anchored bilaterally with percutaneous wires to the infraorbital rims and the pyriform apertures (gauge of 0.4 or $0.5 \mathrm{~mm}$ of stainless steel wire). When a Le Fort I osteotomy $(n=3)$ was performed, the RED was anchored bilaterally with percutaneous wires to the pyriform apertures only. In both cases the use of plates or screws were unnecessary, as the percutaneous wire osteosynthesis was fixed directly to the bone.

All patients were operated on by one surgeon.

\section{Surgical procedure}

All surgeries were performed in an operating room, under general anaesthesia, using orotracheal intubation. Through a maxillary vestibular approach, a Le Fort I $(n=3)$ or III $(n=6)$ osteotomy was performed using a reciprocating or piezoelectric saw. In patients who underwent a Le Fort III osteotomy a transconjuctival approach was also utilised. A nasal septum osteotomy and pterygomaxillary disjunction were performed subsequently.

A cylindrical burr was used to perforate below the pyriform apertures. A $0.40-\mathrm{mm}$ stainless steel wire was passed through the apertures to the floor of the nasal cavity. A 14-G cannula was used to move the wire percutaneously towards the skin along the nasolabial fold.

Patients who underwent a Le Fort III osteotomy also underwent upper skeletal anchorage. An aperture was opened with a cylindrical burr alongside the mid lateral 
Table 1: Patient distribution according to age, gender, diagnosis, type of osteotomy, amount of distraction, relapse, and follow-up

\begin{tabular}{|c|c|c|c|c|c|c|c|c|c|}
\hline $\begin{array}{l}\text { Patients } \\
\text { No. }\end{array}$ & $\begin{array}{c}\text { Age } \\
\text { (years) }\end{array}$ & Gender & Diagnosis & $\begin{array}{l}\text { Overjet before } \\
\text { surgery }\end{array}$ & $\begin{array}{l}\text { Type of } \\
\text { osteotomy }\end{array}$ & $\begin{array}{c}\text { Days of } \\
\text { activation }(\mathrm{mm})\end{array}$ & $\begin{array}{l}\text { Overjet a year after } \\
\text { distraction }(\mathrm{mm})\end{array}$ & $\begin{array}{l}\text { Relapse } \\
(\mathrm{mm})\end{array}$ & $\begin{array}{l}\text { Follow-up } \\
\text { (months) }\end{array}$ \\
\hline 1 & 9 & $\mathrm{M}$ & Crouzon Sd & -20 & Le Fort III & 30 & +3 & 0 & 15 \\
\hline 2 & 15 & $F$ & $\begin{array}{l}\text { Severe class III } \\
\text { maloclussion }\end{array}$ & -16 & Le Fort III & 25 & +2 & 0 & 55 \\
\hline 3 & 17 & $M$ & Crouzon Sd & -13 & Le Fort III & 24 & +3 & 0 & 24 \\
\hline 4 & 25 & M & Crouzon Sd & -16 & Le Fort III & 22 & +3 & 0 & 36 \\
\hline 5 & 9 & M & CLP & -15 & Le Fort III & 25 & -5 & 5 & 60 \\
\hline 6 & 20 & $\mathrm{~F}$ & CLP & -12 & Le Fort I & 20 & +2 & 0 & 30 \\
\hline 7 & 18 & M & Crouzon Sd & -25 & Le Fort III & 30 & +2 & 0 & 36 \\
\hline 8 & 21 & $\mathrm{~F}$ & CLP & -14 & Le Fort I & 25 & +3 & 0 & 36 \\
\hline 9 & 24 & $M$ & CLP & -12 & Le Fort I & 20 & +2 & 0 & 30 \\
\hline Average & 17.5 & & & -15.8 & & 24.5 & 1.6 & 0.55 & 35.7 \\
\hline
\end{tabular}

CLP: cleft lip and palate; Sd: syndrome

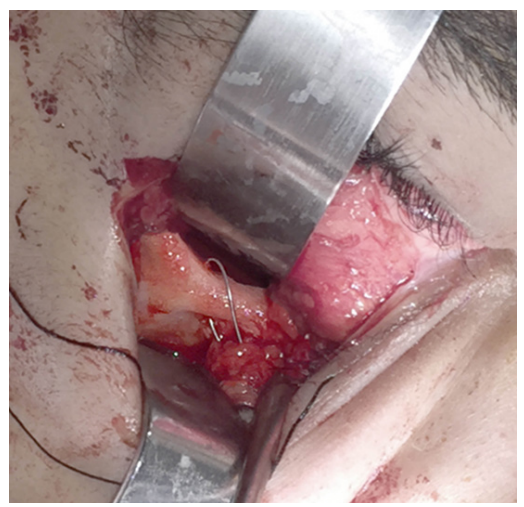

Figure 1: Wires skeletally fixed to the infra orbital rim

area of the infraorbital rims over the anterior wall of the maxilla. Through this aperture a $0.40-\mathrm{mm}$ stainless steel wire was passed through and returned through the osteotomy on the orbit's floors. A 14-G cannula was used as a guide to move the wires towards the skin, allowing a percutaneous exit [Figure 1].

A halo frame was fixed to the skull using three percutaneous screws on each side, secured to the scalp. The screws were parallel to Frankfort's horizontal plane.

The distraction vector was calculated according to the patients' needs. The latency period was one day. Active distraction started on the first postoperative day at a rate of $1.0 \mathrm{~mm}$ per day divided in $0.5 \mathrm{~mm}$ in the morning and $0.5 \mathrm{~mm}$ in the evening. In every case desired advancement was achieved without overcorrection. The distraction device was removed after a consolidation period of 6 to 8 weeks, followed by intermaxillary elastics to improve the occlusal relationship.

The RED was removed in an outpatient care room without the need for local anaesthetic. The skeletal anchor wires were cut and removed The halo frame's

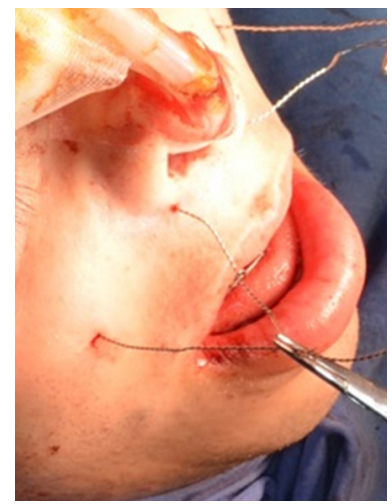

Figure 2: Wires emerging percutaneously. They are fixed to the infraorbital rim and the piriform aperture

pins were unscrewed and removed.

\section{RESULTS}

On average, a $24.0 \pm 3.6 \mathrm{~mm}$ (range $20-30 \mathrm{~mm}$ ) midface distraction was obtained. The overjet before surgery was on average $-15.8 \pm 4.2 \mathrm{~mm}$, and a year after distraction was $1.6 \pm 2.5 \mathrm{~mm}$ [Table 1]. None of the patients reported complications during the course of the treatment. There were incidents of the wires breaking, skin infection or bone fractures associated with the skeletal anchors.

The distraction objective was achieved in all but one patient, who suffered a relapse of $5 \mathrm{~mm}$. The deformity was secondary to a cleft lip and palate, and the patient did not have ideal dental overjet or overbite in handarticulated models before the treatment. The patient subsequently underwent a conventional Le Fort I osteotomy to achieve ideal results.

Patient follow-up lasted 35.7 months on average, ranging between 15 and 60 months. It is vital to highlight that follow-up continues [Figures 2-4]. 


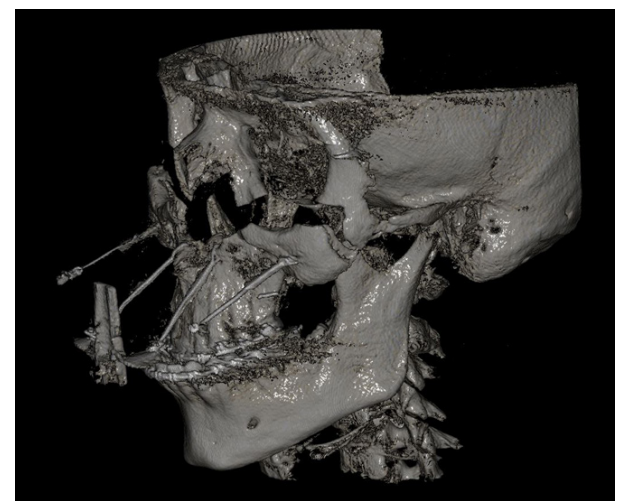

Figure 3: Cone beam computed tomography showing the wires anchored directly to the bone

\section{DISCUSSION}

Distraction osteogenesis was introduced in the craniofacial field in 1992 by McCarthy et al. ${ }^{[13]}$ to correct mandibular hypoplasia. ${ }^{[14]}$ The procedure has since been widely used in the field of craniofacial surgery, and is considered today as an alternative method to treating craniofacial dysplasia. ${ }^{[5,15]}$ Intra and extraoral distraction devices can be used. Extraoral devices are easier to handle, allow for more force to be applied and for greater advancement to be achieved. They also allow modification and better control of the distraction vector. ${ }^{[6,15,16]}$ When an extraoral device is used, further surgery is not needed to remove the distractor. ${ }^{[6,17]}$
This study aimed to overcome some of these limitations by modifying the method by which facial bones are anchored to an extraoral distraction device, specifically RED II. Several authors ${ }^{[1,5,10,11]}$ describe one of the limitations being the need for teeth to be used as anchorage. Regardless of the dentition phase, they need to be in good and healthy condition. In the proposed technique, the pyriform apertures and infraorbital rims are used as anchorage points and teeth are only necessary to stabilize the distracted segments once they have achieved the desired occlusion.

Nevertheless, as Nishimoto et al. ${ }^{[18]}$ emphasised, the presence of teeth is ideal because it diminishes the chance of relapse, since occlusion holds skeletal bases in position. Furthermore, they state that when teeth are missing, consolidation time should be longer.

In the publication by Nout et al. ${ }^{[10]}$ an alternative is mentioned for distraction with RED without dental anchoring in a patient diagnosed with Pfeiffer's syndrome. They suggested using bilateral anchorage to the pyriform aperture only, fixed with screws. SARED does not require the use of osteosynthesis (plates nor screws) nor a custom-made intraoral orthodontic splint, reducing the cost of treatment and diminishing the risk of damage of dental follicles and roots.

Since teeth anchoring is unnecessary in SARED, the force can be applied directly to the bone. This in turn
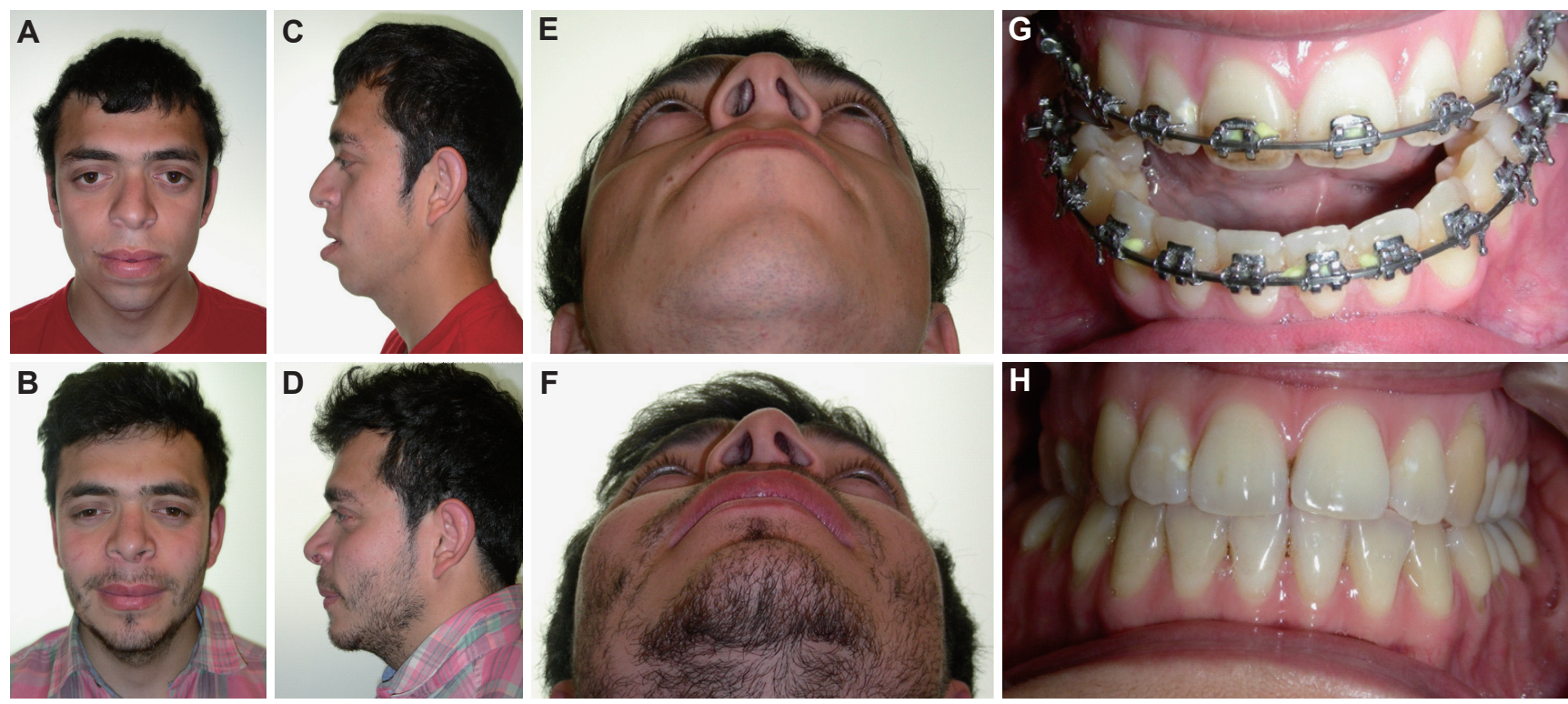

Figure 4: Patient 4. (A) Frontal view before surgery. Crouzon syndrome patient with severe midface hypoplasia; (B) frontal view after surgery. Note the reduction in exophthalmos; (C) lateral view before surgery. Hypoplastic maxilla, exophtalmos due to shallow eye sockets, relative mandibular prognathism; (D) lateral view after surgery. Adequate advancement of the maxilla, reduced exophtalmos; (E) axial view before surgery. Note exophtalmos due to shallow eye sockets and the asymmetric nostrils; $(F)$ axial view after surgery. The advancement of the maxilla with an adequate cheekbone and infraorbital rim projection; (G) occlusal view before surgery. Negative overjet showing the discrepancy between the maxilla and the mandible teeth; $(\mathrm{H})$ occlusal view after surgery. Adequate occlusion achieved with midface distraction osteogenesis 
means that the line of action of the force vector is direct as there is no intermediary device to reduce the force. As Figueroa and Polley ${ }^{[5]}$ discuss, the wire splint of RED I, which has an extraoral wire and traction hooks, has some flexibility, which allows the distraction wire force to accumulate and then be liberated gradually. In other words, once the screw is activated, the distraction effect is not immediate, but as the wire recovers its shape it becomes a continuous tension. ${ }^{[3,11,19]}$ We believe the mechanism described by the authors ${ }^{[5]}$ is not ideal because the wire undergoes elastic deformity that prevents the applied force from being transferred in its entirety to the bone being distracted. ${ }^{[11,12]}$

SARED is more comfortable than the intraoral splint anchorage, because it does not interfere with the functions of the oral cavity. It allows uninterrupted eating because there is no device, wires or splints inside the mouth. Patients can, therefore, brush their teeth even when they have braces. In addition, patients with SARED can continue their orthodontic treatment during the whole distraction and consolidation period.

The percutaneous exits of the wires from the nasolabial folds do not compromise aesthetics because the scars are hidden in face wrinkles. Other authors have previously suggested the use of percutaneous wires emerging from the zygomatic bone to distract with RED II and have reported no major complications. ${ }^{[18]}$

Finally, SARED does not require further surgery to be removed, as do osteosynthesis anchored devices. ${ }^{[11]}$ The halo and wires can be pulled out without local anaesthetic on an outpatient basis. The use of SARED simplifies a process that is highly complex itself and delivers better conditions for the patient and surgeon.

Using SARED is a convenient method to distract the midface. Advantages include better control of the distraction vector and force when compared to the use of a dental anchorage (RED I) or a dental anchorage with an osteosynthesis plate anchorage (RED II). SARED improves patient comfort levels, oral hygiene and overall oral health. The method proposed is simple, reduces costs and complications.

\section{DECLARATIONS}

\section{Authors' contributions}

Concept, design, definition of intellectual content, literature search, clinical studies, data acquisition, data analysis, statistical analysis, manuscript preparation, manuscript editing, and manuscript review: R. Fariña, F. Salinas
Financial support and sponsorship

None.

\section{Conflicts of interest}

The authors have no commercial or financial associations that might create a conflict of interest with the information presented in this manuscript.

\section{Patient consent}

All patients signed an informed consent letter before participating in the study.

\section{Ethics approval}

This study was approved by ethical board of Hospital del Salvador.

\section{REFERENCES}

1. Polley JW, Figueroa AA. Rigid external distraction: its application in cleft maxillary deformities. Plast Reconstr Surg 1998;102:1360-72; discussion 1373-4.

2. Polley JW, Figueroa AA. Management of severe maxillary deficiency in childhood and adolescence through distraction osteogenesis with an external, adjustable, rigid distraction device. J Craniofac Surg 1997;8:181-5; discussion 186.

3. Molina F, Monasterio FO, de la Paz Aguilar M, Barrera J. Maxillary distraction: aesthetic and functional benefits in cleft lip-palate and prognathic patients during mixed dentition. Plast Reconstr Surg 1998;101:951-63.

4. Combs PD, Harshbarger RJ 3rd. Le fort I maxillary advancement using distraction osteogenesis. Semin Plast Surg 2014;28:193-8.

5. Figueroa AA, Polley JW. Management of severe cleft maxillary deficiency with distraction osteogenesis: procedure and results. Am J Orthod Dentofacial Orthop 1999;115:1-12.

6. Burstein F, Soldanska M, Granger M, Berhane C, Schoemann M. Initial experience with a new intraoral midface distraction device. $J$ Craniofac Surg 2015;26:1224-8.

7. Nishimoto S, Oyama T, Shimizu F, Tsugawa T, Nagashima T, Yamamoto K, Kamiji T, Kanomi R. Fronto-facial monobloc advancement with rigid external distraction (RED-II) system. $J$ Craniofac Surg 2004; 15:54-9.

8. Miyazaki H, Katada H, Ichinokawa Y, Hirabayashi S, Sueishi K. Orthodontic treatment in combination with Le Fort II bone distraction in patient with Apert syndrome. Bull Tokyo Dent Coll 2013;54:9-17.

9. Raposo-Amaral CE, Denadai R, Ghizoni E, Buzzo CL, RaposoAmaral CA. Family of crouzon syndrome represents the evolution of the frontofacial monobloc advancement technique: from immediate movement to monobloc distraction to monobloc bipartition distraction. J Craniofac Surg 2015;26:1940-3.

10. Nout E, Cesteleyn LL, van der Wal KG, van Adrichem LN, Mathijssen IM, Wolvius EB. Advancement of the midface, from conventional Le Fort III osteotomy to Le Fort III distraction: review of the literature. Int J Oral Maxillofac Surg 2008;37:781-9.

11. Baek SH, Seo YJ. Application of orthodontic mini-implants and ligation for absolute skeletal anchorage to the intraoral labiolingual appliance: midface distraction osteogenesis cases treated with the RED System. J Craniofac Surg 2011;22:609-13.

12. Resnick CM, Rottgers SA, Langenfeld CC, Mulliken JB, Padwa BL. Novel rigid external distraction device improves stability and 
controls the vector during midfacial advancement. J Craniofac Surg 2016;27:1056-9.

13. McCarthy JG, Schreiber J, Karp N, Thorne CH, Grayson BH. Lengthening the human mandible by gradual distraction. Plast Reconstr Surg 1992;89:1-8; discussion 9-10.

14. Figueroa AA, Polley JW, Figueroa AD. Biomechanical considerations for distraction of the monobloc, Le Fort III, and Le Fort I segments. Plast Reconstr Surg 2010;126:1005-13.

15. Tong H, Liu L, Tang X, Song T, Yin N, Zhang Z, Zhao Z. Midface distraction osteogenesis using a modified external device with elastic distraction for crouzon syndrome. J Craniofac Surg 2017; DOI: 10.1097/SCS.0000000000003377

16. Fernández-Olarte $\mathrm{H}$, Gómez-Delgado A, Varón-Cardona D,
Trujillo-Saldarriaga S. Combination of transconjunctival and lateral canthotomy with modified endaural approaches in midface osteogenic distraction. Br J Oral Maxillofac Surg 2016;54:707-9.

17. Nout E, Wolvius EB, van Adrichem LN, Ongkosuwito EM, van der Wal KG. Complications in maxillary distraction using the RED II device: a retrospective analysis of 21 patients. Int J Oral Maxillofac Surg 2006;35:897-902.

18. Nishimoto S, Oyama $\mathrm{T}$, Shimizu F, Tsugawa $\mathrm{T}$, Nagashima $\mathrm{T}$, Yamamoto K, Kamiji T, Kanomi R. Fronto-facial monobloc advancement with rigid external distraction (RED-II) system. $J$ Craniofac Surg 2004;15:54-9.

19. Patel N, Fearon JA. Treatment of the syndromic midface: a long-term assessment at skeletal maturity. Plast Reconstr Surg 2015;135:e731-42. 